\title{
BYPASS1-LIKE, A DUF793 Family Protein, Participates in Freezing Tolerance via the CBF Pathway in Arabidopsis
}

Tao Chen ${ }^{1}$, Jia-Hui Chen ${ }^{1}$, Wei Zhang ${ }^{1}$, Gang Yang ${ }^{1}$, Li-Juan Yu', Dong-Ming Lí ${ }^{2}$, Bo Li ${ }^{1}$, Hong-Mei Sheng ${ }^{1}$, Hua Zhang ${ }^{1 *}$ and Li-Zhe An ${ }^{1,3 *}$

'School of Life Sciences, The Key Laboratory of Cell Activities and Stress Adaptations, Ministry of Education, Lanzhou University, Lanzhou, China, ${ }^{2}$ College of Life Sciences, Zhejiang University, Hangzhou, China, ${ }^{3}$ School of Forestry, Beijing Forestry University, Beijing, China

OPEN ACCESS

Edited by:

William Walter Adams III, University of Colorado Boulder, United States

Reviewed by:

Jian-Kang Zhu,

Purdue University, United States

Timothy Artlip,

Appalachian Fruit Research Station (ARS-USDA),

United States

${ }^{*}$ Correspondence:

Hua Zhang

zhanghua@/zu.edu.cn

Li-Zhe An

lizhean@/zu.edu.cn

Specialty section: This article was submitted to Plant Abiotic Stress, a section of the journal Frontiers in Plant Science

Received: 24 April 2019 Accepted: 05 June 2019 Published: 26 June 2019

Citation:

Chen T, Chen J-H, Zhang W, Yang G, Yu L-J, Li D-M, Li B, Sheng H-M, Zhang $H$ and An L-Z (2019) BYPASS1-LIKE, A DUF793 Family Protein, Participates in Freezing Tolerance via the $C B F$ Pathway in Arabidopsis. Front. Plant Sci. 10:807. doi: 10.3389/fp/s.2019.00807
The C-REPEAT BINDING FACTOR signaling pathway is strictly modulated by numerous factors and is essential in the cold response of plants. Here, we show that the DUF793 family gene BYPASS1-LIKE modulates freezing tolerance through the CBFs in Arabidopsis. The expression of $B 1 L$ was rapidly induced under cold treatment. Comparing to wild type, $B 1 L$ knockout mutants were more sensitive to freezing treatment, whereas $\mathrm{B} 1 \mathrm{~L}-$ overexpressing lines were more tolerant. The expression of $\mathrm{CBF}$ and $\mathrm{CBF}$ target genes was significantly decreased in b1/ mutant. Using yeast two-hybrid screening system, 14-3-3 $\lambda$ was identified as one of proteins interacting with B1L. The interaction was confirmed with bimolecular fluorescence complementation assay and co-immunoprecipitation assay. Biochemical assays revealed that $b 1 /$ mutation promoted the degradation of CBF3 compared to wild type, whereas $14-3-3 \kappa \lambda$ mutant and $b 1 / 14-3-3 \kappa \lambda$ mutant suppressed

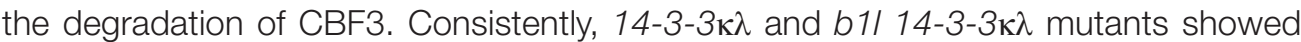
enhanced freezing tolerance compared to wild type. These results indicate that $\mathrm{B} 1 \mathrm{~L}$ enhances the freezing tolerance of plants, at least partly through stabilizing CBF. Our findings improve our understanding of the regulation of $\mathrm{CBF}$ in response to cold stress.

Keywords: 14-3-3 proteins, Arabidopsis, CBF pathway, cold stress, DUF793, protein degradation

\section{INTRODUCTION}

Temperature is one of the most important environmental factors that affect the survival, growth, and reproduction of plants. Plants adapt to freezing stress through multiple physiological and biochemical processes. After exposure to low temperatures above freezing, temperate plants acquire freezing tolerance, a process that is termed cold acclimation (Guy, 1990; Thomashow, 1999). The expression of a class of APETALA2/ETHYLENE-RESPONSIVE FACTOR (AP2/ERF) transcription factor, C-REPEAT BINDING FACTOR/DROUGHT RESPONSE ELEMENT BINDING FACTOR $1 B$ (CBF/DREB1), is rapidly induced under cold stress, playing a central role in the cold acclimation of Arabidopsis (Stockinger et al., 1997; Liu et al., 1998; Thomashow, 1999). CBFs bind to the CRT/DRE element of COLD-REGULATED (COR) genes, inducing their expression and conferring an enhanced freezing tolerance (Yamaguchi-Shinozaki and Shinozaki, 1994; 
Stockinger et al., 1997; Gilmour et al., 1998; Liu et al., 1998; Thomashow, 1999).

The expression of $C B F s$ is regulated by numerous transcription factors. Using CBF3-LUC transgenic plants, Chinnusamy et al. identified a basic helix-loop-helix (bHLH) transcription factor named INDUCER OF CBF EXPRESSION 1 (ICE1; Chinnusamy et al., 2003). ICE1 promotes the expression of CBF3 through the binding to $M Y C$ cis-elements within the promoter region of CBF3 (Chinnusamy et al., 2003). BRASSINAZOLERESISTANT 1 (BZR1), LATE ELONGATED HYPOCOTYL (LHY), and CALMODULIN-BINDING TRANSCRIPTION ACTIVATOR 3 (CAMTA3) were also found to positively regulate the expression of CBFs (Doherty et al., 2009; Dong et al., 2011; Li et al., 2017a,b). On the other hand, MYB15, ETHYLENEINSENSITIVE 3 (EIN3), and PHYTOCHROME-INTERACTING FACTOR 3 (PIF3) repress CBF expression (Agarwal et al., 2006; Shi et al., 2012; Jiang et al., 2017).

The posttranslational regulation of $\mathrm{CBF}$ is also involved in the plant response to cold stress (Liu et al., 2017; Ding et al., 2018). In this process, 14-3-3 proteins are phosphorylated by COLD-RESPONSIVE PROTEIN KINASE 1 (CRPK1) and translocated from the cytoplasm to the nucleus, where 14-3-3 proteins can interact with $\mathrm{CBFs}$ and trigger the degradation of CBFs through the 26S proteasome pathway (Liu et al., 2017). By contrast, BTF3-LIKE (BTF3L) inhibits the degradation of CBFs by interacting with CBF proteins (Ding et al., 2018). However, the proteins that negatively modulate the

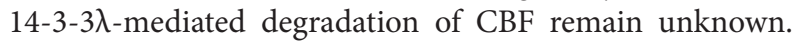

In Arabidopsis, at least 12 proteins contain a conserved DUF793 domain, but only few members have been functionally characterized. BYPASS1 (BPS1) is required to produce a rootsourced signal that moves to the shoot and arrests growth of shoot, through modification of cytokinin signaling (Van Norman et al., 2004, 2011; Lee et al., 2016). ROH1 interacts with the exocyst subunit EXO70A1 and is involved in the localized deposition of seed coat pectin (Kulich et al., 2010). At1g74450 gene affects the plant height, pollen development, and composition of the inner seed coat mucilage layer (Visscher et al., 2015). AT1G18740, which we named as BYPASS1-LIKE (B1L), and AT1G74450 are both responsive to multiple abiotic stresses (Ma and Bohnert, 2007). However, more insight into the molecular function of the DUF793 proteins is in need.

In this study, we found that $B 1 L$, which is rapidly induced under cold treatment, modulates freezing tolerance through the CBFs in Arabidopsis. To be specific, B1L reduces the

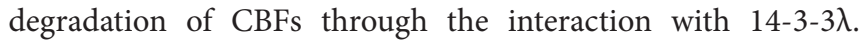
Our results indicate that $\mathrm{B} 1 \mathrm{~L}$ positively modulates plant freezing tolerance, at least partly through stabilizing CBFs.

\section{MATERIALS AND METHODS}

\section{Plant Materials and Growth Conditions}

Arabidopsis thaliana Col-0 was used as the wild type. The mutant and transgenic lines that were used in this study were

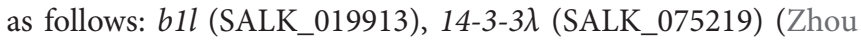
et al., 2014; Liu et al., 2017), 14-3-3א (SALK_148929)

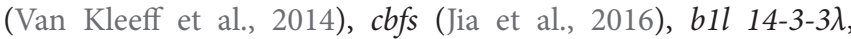

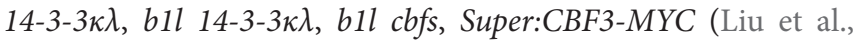
2017), Super:CBF3-MYC/b1l, B1L b1l \#1, B1L b1l \#2, B1L-OE \#1, B1L-OE\#2, ProB1L:B1L-GFP \#1, and ProB1L:GUS.

b1l was obtained from ABRC. $14-3-3 \kappa \lambda$ was generated by crossing $14-3-3 \lambda$ and $14-3-3 \kappa .14-3-3 \lambda, 14-3-3 \kappa$, and $14-3-3 \kappa \lambda$ were kindly provided by the Li Jia laboratory of Lanzhou University. cbfs and Super:CBF3-MYC were kindly provided by the Shu-Hua Yang laboratory from the China Agricultural

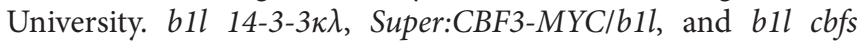
were generated through genetic crossing.

The ProB1L:B1L-3×FLAG fusion and the $b 1 l$ restored plants (B1L b1l \#1 and \#2) were obtained via amplifying the B1L genomic region, including the 2000-bp promoter fragment, and cloning the resulting PCR product into the pMDC302 Gateway binary vector. The ProB1L:B1L-GFP transgenic plants (ProB1L:B1L-GFP \#1) were obtained by amplifying the same genomic region and cloning it into the pMDC107 Gateway binary vector. The ProB1L:GUS transgenic plants were obtained by amplifying the ProB1L fragment and cloning it into the pBIB-GUS vector. The 35S:YFP-B1L fusion and the B1L-overexpressing transgenic lines (B1L-OE \#1 and \#2) were obtained by amplifying B1L cDNA and cloning the resulting PCR product into the pEarlygate 104 Gateway binary vector.

Plants were grown at $22^{\circ} \mathrm{C}$ under long-day conditions $(16 \mathrm{~h}$ light $/ 8 \mathrm{~h}$ dark) in soil or agar plates $(1 / 2 \mathrm{MS}, 1 \%$ sucrose, and $0.8 \%$ agar).

All primer sequences that were used in this study are listed in Supplementary Table S1.

\section{Plant Freezing Assay}

The plant freezing assays were performed as previously described (Zhu et al., 2004; Miura et al., 2007) with modifications. Plants were grown in soil at $21^{\circ} \mathrm{C}$ under long-day (LD) conditions for 3 weeks before the treatments were performed. For each line, the plant freezing assay was performed with four pots of 16 plants. For the treatments without cold acclimation, the pots with different plants were alternately placed in a controlledtemperature chamber (MIR-254; SANYO) for approximately $30 \mathrm{~min}$ at $0^{\circ} \mathrm{C}$ and then for $1 \mathrm{~h}$ at $0^{\circ} \mathrm{C}$ before the temperature was decreased by $1^{\circ} \mathrm{C} / \mathrm{h}$. The final desired sub-zero temperature was maintained for the indicated period before the temperature was again increased to $4^{\circ} \mathrm{C}$. The plants were then kept at $4^{\circ} \mathrm{C}$ for $12 \mathrm{~h}$ before they were returned to $21^{\circ} \mathrm{C}$. Survival was scored 5 days later, and those plants able to maintain a green color at the shoot apex were counted as survivors. For the cold acclimation experiments, 3-week-old plants were acclimated to $4^{\circ} \mathrm{C}$ in the light for 3 days. The freezing treatment was then performed in the same manner as that for the non-acclimated plants, with the final desired freezing temperature maintained for the indicated period. The experiments were conducted with three independent biological replicates.

\section{Electrolyte Leakage Assay}

Electrolyte leakage of detached leaves from 3-week-old plants was measured as previously described (Zhao et al., 2017) 
with modifications. Plants were grown in soil at $21^{\circ} \mathrm{C}$ in $\mathrm{LD}$ conditions, and the fifth oldest leaf was used. The leaves were placed in tubes containing $100 \mu \mathrm{l}$ of deionized water. Ice chips were added to the tubes, and the tubes were kept at $0^{\circ} \mathrm{C}$ for $30 \mathrm{~min}$, followed by a temperature decrease of $1^{\circ} \mathrm{C} / \mathrm{h}$. The samples were removed at the indicated temperature points and immediately placed on ice. Ten milliliters of deionized water were added to each tube, and the samples were incubated for $1 \mathrm{~h}$ at $21^{\circ} \mathrm{C}$ under gentle shaking, after which the conductivity of the solution was determined via a conductivity meter (DDSJ$308 \mathrm{~A}$; INESA). The tubes were then autoclaved at $120^{\circ} \mathrm{C}$ for $30 \mathrm{~min}$, and the conductivity of the solution was measured again after the samples were cooled to $21^{\circ} \mathrm{C}$. Electrolyte leakage was quantified as a percentage of the conductivity after the treatment relative to total conductivity. The experiments were conducted with three independent biological replicates.

\section{qRT-PCR and RT-PCR Assays}

Total RNA was extracted with a RNAprep pure plant kit (TIANGEN) and treated with DNaseI to digest the DNA. Firststrand cDNA was synthesized from $1 \mu \mathrm{g}$ of RNA using the RevertAid First Strand cDNA Synthesis Kit (Thermo Scientific) according to the manufacturer's instructions.

For the qRT-PCR assay, 12-day-old seedlings were grown on agar plates and treated at $4^{\circ} \mathrm{C}$ in the light, and the plant material was collected in a time-course manner. qRT-PCRs were performed with the SsoFast EvaGreen Supermix (BioRad) using the CFX96 Real-Time System (Bio-Rad). Actin2/8 was used for the normalization of the results (Shi et al., 2012; Liu et al., 2017; Li et al., 2017a). qRT-PCRs were typically performed with at least three independent biological samples, and each was measured with at least three technical repeats. The statistical significance of the differences between two samples was assessed using a Student's $t$ test.

For the RT-PCR analysis, the roots and whole seedlings were collected from 12-day-old seedlings; the leaves and stems were collected from 5-week-old plants; and the flowers and siliques were collected from 8 -week-old plants. $\beta$-TUBULIN was used as an internal control. Ethidium bromide staining was used to detect the PCR products.

\section{Confocal Microscopic Analysis}

B1L cDNA was amplified and cloned into the pEarleygate104 Gateway binary vector. The plasmid was introduced into Agrobacterium tumefaciens GV3101 and transiently expressed in $N$. benthamiana leaves. Two days after infiltration, the YFP fluorescence signal was detected with a confocal microscope (Leica SP8). The 5-day-old ProB1L:B1L-GFP and B1L-OE with YFP tag seedlings were also used for a subcellular localization assay.

\section{Histochemical GUS Reporter Gene Expression Analysis}

The GUS staining assay was performed as previously described (Chen et al., 2016) with T3 ProB1L:GUS transgenic plants of 1-day-old seedlings, 2-day-old seedlings, 10-day-old seedlings, and 8-week-old mature plants. After staining, the samples were rinsed with acetic acid/methanol [1:3 (v/v)]. The images were collected on a stereomicroscope (Nikon SMZ800).

\section{Y2H Screening and Assay}

AH109 was used as a host strain. B1L cDNA was subcloned into the pGBKT7 Gateway binary vector. The transcriptional activation of B1L-pGBKT7 was detected, and B1L-pGBKT7 was used as bait to screen an Arabidopsis thaliana cDNA library. The transformation was performed according to the Clontech Yeast Protocols Handbook (PT3024), with selection on media lacking leucine (Leu), tryptophan (Trp), histidine (His), and adenine (Ade). The positive clones were isolated and sequenced. To determine the interaction between $B 1 L$ and $14-3-3 \lambda$ in yeast, $B 1 L-\mathrm{N}$ terminal, $B 1 L-\mathrm{C}$ terminal, $B 1 L^{\mathrm{S} 213 \mathrm{~A}}$, and $B 1 L^{\mathrm{S} 213 \mathrm{D}}$ were amplified and cloned into the pGBKT7 Gateway binary vector. The coding sequence of $14-3-3 \lambda$ was amplified and cloned into the pGADT7 Gateway binary vector. The coding sequence of 14-3-3 $\psi$ was also amplified and cloned into the pGADT7 Gateway binary vector to analyze the interaction between B1L and 14-3-3\%. The yeast transformation and growth assays were performed as described above.

\section{BiFC Assay}

To determine the interaction between $\mathrm{B} 1 \mathrm{~L}$ and $14-3-3 \lambda, B 1 L$ cDNA, $B 1 L^{\text {S213A }}$, and $B 1 L^{\text {S213D }}$ were amplified and cloned into PNYFP-X, and 14-3-3 $\lambda$ cDNA was amplified and cloned into the PCCFP-X Gateway binary vector. Plasmids containing $\mathrm{YFP}^{\mathrm{N}}-\mathrm{B} 1 \mathrm{~L}$ and $\mathrm{YFP}^{\mathrm{C}}-14-3-3 \lambda, \mathrm{YFP}^{\mathrm{N}}-\mathrm{B} 1 \mathrm{~L}$ and $\mathrm{YFP}^{\mathrm{C}}, \mathrm{YFP}^{\mathrm{N}}$ and $\mathrm{YFP}^{\mathrm{C}}-14-3-3 \lambda, \mathrm{YFP}^{\mathrm{N}}-\mathrm{B} 1 \mathrm{~L}^{\mathrm{S} 213 \mathrm{D}}$ and $\mathrm{YFP}^{\mathrm{C}}-14-3-3 \lambda$, or $\mathrm{YFP}^{\mathrm{N}}-\mathrm{B} 1 \mathrm{~L}^{\mathrm{S} 213 \mathrm{~A}}$ and $\mathrm{YFP}^{\mathrm{C}}-14-3-3 \lambda$ were introduced into $A$. tumefaciens $\mathrm{GV} 3101$ and transformed to $N$. benthamiana leaves. Two days after infiltration, the YFP fluorescence signal was detected using a Leica SP8 confocal microscope.

\section{CoIP Assay}

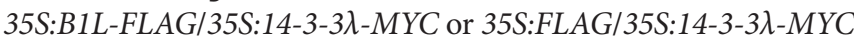
were co-expressed into $N$. benthamiana leaves. Total protein was subsequently extracted in IP buffer containing $50 \mathrm{mM}$ Tris-HCl, pH 7.6; 150 mM NaCl; 10\% glycerol; and 1× Cocktail. The cell debris were removed via two 12-min centrifugations at $16,000 \mathrm{~g}$ at $4^{\circ} \mathrm{C}$. The supernatant was collected and incubated with anti-FLAG agarose (Abmart) overnight at $4^{\circ} \mathrm{C}$. After washing with IP buffer five times, the co-immunoprecipitated products were separated by SDS-PAGE and detected with anti-MYC (1:5,000, Abcam) and anti-FLAG (1:10,000, Abmart) antibodies.

\section{Protein Degradation Assay}

Protein degradation assays were performed as previously described (Wang et al., 2009; Liu et al., 2010) with modifications. Arabidopsis seedlings were harvested and ground to a fine powder in liquid nitrogen. Total protein was subsequently extracted in degradation buffer containing $25 \mathrm{mM}$ Tris- $\mathrm{HCl}$, $\mathrm{pH} 7.5 ; 10 \mathrm{mM} \mathrm{NaCl} ; 10 \mathrm{mM} \mathrm{MgCl}$; $5 \mathrm{mM} \mathrm{DTT}$; and $1 \times$ Cocktail, and the protein concentration was determined. 
For the cell-free degradation assay, $100 \mu \mathrm{g}$ purified MBP-CBF3 recombinant proteins were incubated with the total proteins that were extracted from wild type, $b 1 l$, B1L-OE \#1, 14-3-3k $\lambda$, or b1l $14-3-3 \kappa \lambda$ plants in the presence of $1 \mathrm{mM}$ ATP (Sigma) at $25^{\circ} \mathrm{C}$ for different time courses, and the CBF3-MBP proteins were detected via immunoblotting with an anti-MBP antibody (1:10,000; Abcam). MG132 (Sigma) was added to the various degradation assays, as indicated.

\section{Software Availability}

Band intensity quantifications of CBF3 were performed using the Image ${ }^{1}$. The motifs within $\mathrm{B} 1 \mathrm{~L}$ protein that are likely to be phosphorylated and bound by 14-3-3 proteins were predicted with Scansite $4^{2}$. The diagram for the B1L protein was performed using the $\mathrm{IBS}^{3}$.

\section{ACCESSION NUMBERS}

Sequence data from this article can be found in the Arabidopsis Genome Initiative or GenBank/EMBL/Swiss-Prot databases under the following accession numbers: B1L (AT1G18740), CBF1 (AT4G25490), CBF2 (AT4G25470), CBF3 (AT4G25480), COR15a (AT2G42540), COR15b (AT2G42530), COR47 (AT1G20440), RD29A (AT5G52310), ERF4(AT3G15210), ERF11(AT1G28370),

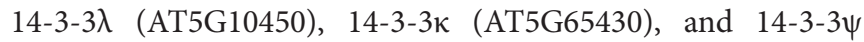
(AT5G38480).

\section{RESULTS}

\section{Cold Induces the Expression of B1L, Which Positively Modulates Freezing Tolerance}

To investigate the function of B1L in plant freezing tolerance, a T-DNA insertion mutant $(b 1 l)$ was obtained from the Arabidopsis Biological Resource Center (ABRC), and the complete disruption of $B 1 L$ expression in the $b 1 l$ mutant was verified (Supplementary Figure S1A). Three-week-old plants showing similar growth and development in wild type and $b 1 l$ were used to examine the freezing tolerance (Supplementary Figure S1B). The b1l mutant was more sensitive to freezing treatment than wild type under cold-acclimated (CA) conditions (Figures 1A,B). ProB1L:B1L$3 \times F L A G$ was then transformed to b1l mutant, and the complementation lines $\left(\begin{array}{lll}B 1 L & b 1 l\end{array}\right)$ fully restored the freezing sensitivity of b1l (Supplementary Figure S2). Transgenic plants overexpressing B1L-YFP (B1L-OE) were also used to examine the role of B1L (Supplementary Figure S3). B1L-OE \#1 and \#2 plants were more freezing tolerant compared to wild type, particularly under non-acclimated (NA) conditions (Figures 1C-F). Consistently, ion leakage in the b1l mutant was higher than that in wild type after freezing treatment (Figure 1G), whereas the ion leakage of the B1L-OE \#1 and \#2 plants was lower than

${ }^{1}$ https://imagej.nih.gov/ij/

${ }^{2} \mathrm{http}: / /$ scansite4.mit.edu

${ }^{3}$ http://ibs.biocuckoo.org/download.php that of wild type after freezing treatment (Figure 1H). A quantitative real-time PCR (qRT-PCR) assay was used to investigate the expression of $B 1 L$ under cold treatment $\left(4^{\circ} \mathrm{C}\right)$. The expression of $B 1 L$ quickly increased after $1 \mathrm{~h}$ and reached a peak after $6 \mathrm{~h}$ (Figure 1I). These data indicate that B1L acts as a positive regulator of freezing tolerance in Arabidopsis.

\section{B1L Was Shown to Modulate the Expression of Genes in the CBF Pathway}

To examine whether B1L participates in plant cold acclimation, the expression of CBF pathway genes that perform important roles in cold acclimation was examined through qRT-PCR. The qRT-PCR results showed that both the CBFs and CBF target genes were dramatically reduced in the $b 1 l$ mutants compared to wild type (Figure 2). These results indicate that $\mathrm{B} 1 \mathrm{~L}$ positively regulates the expression of $\mathrm{CBF}$ pathway genes.

\section{B1L Is Expressed in Most Tissues and B1L Protein Localizes to the Cytoplasm and the Nucleus}

To analyze the expression pattern of $B 1 L$, the total RNA from different tissues of wild type was collected and tested. Semiquantitative RT-PCR results showed that B1L was expressed in most tissues; the mRNA from b1l seedlings was used as a negative control (Figure 3A). GUS staining assay using ProB1L:GUS transgenic plants revealed that B1L was predominately expressed in the roots, leaves, and flowers (Figures 3B-F).

To examine the subcellular localization of $\mathrm{B} 1 \mathrm{~L}, \mathrm{~A}$ ProB1L:B1L-GFP construct was generated and transformed to b1l mutant plants (ProB1L:B1L-GFP \#1). Under microscope, GFP fluorescence signal was detected in both the cytoplasm and nucleus (Figure 3G). The transient expression of 35S:YFP-B1L in Nicotiana benthamiana and the stable expression of 35S:YFP-B1L in Arabidopsis were also used to show that B1L localizes to the cytoplasm and nucleus (Supplementary Figure S4).

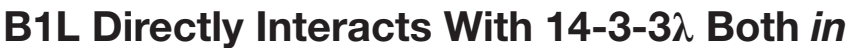 vitro and in vivo}

To further investigate B1L function in the freezing tolerance of plants, a yeast two-hybrid ( $\mathrm{Y} 2 \mathrm{H})$ screening system was used. A 14-3-3 family protein, 14-3-3 $\lambda$, was identified. A bimolecular fluorescence complementation (BiFC) assay was used to confirm the interaction, and the results showed that $\mathrm{B} 1 \mathrm{~L}$ interacts with 14-3-3 $\lambda$ under both cold treatment $\left(4^{\circ} \mathrm{C}, 6 \mathrm{~h}\right)$ and normal conditions (Figure 4A). A co-immunoprecipitation (coIP) assay using co-expressed B1L-FLAG and 14-3-3 $\lambda$-MYC was also performed and the result verified that B1L directly interacts with 14-3-3 $\lambda$ in vivo (Figure 4B).

The 14-3-3 proteins are well-known to bind to many proteins that are phosphorylated by recognizing phosphoserine or phosphothreonine within their conserved binding motifs (Jaspert et al., 2011; Wang et al., 2011; Yoon and Kieber, 2013; Zhou et al., 2014; Huang et al., 2018). To search for 14-3-3 motifs in B1L sequence, the Scansite $4^{4}$ was used to predict

${ }^{4}$ https://scansite4.mit.edu/4.0/ 


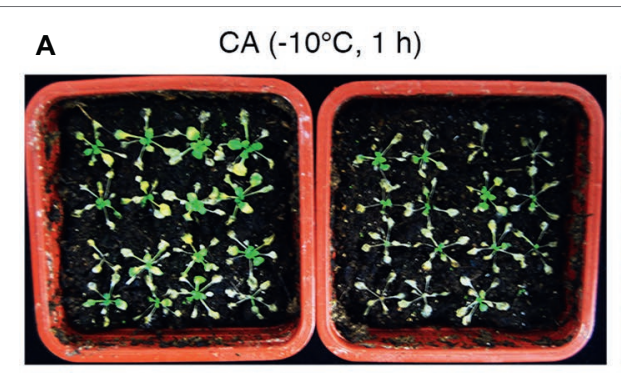

WT

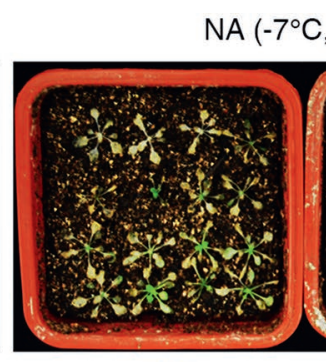

WT

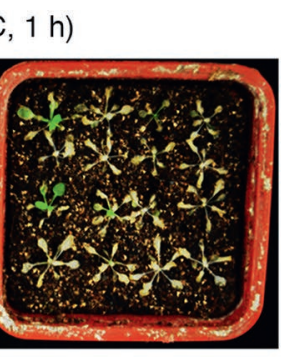

b11

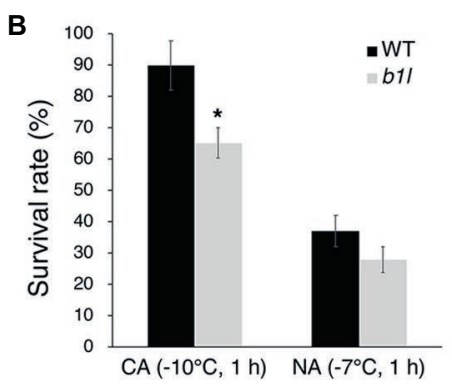

C

$\mathrm{CA}\left(-10^{\circ} \mathrm{C}, 3 \mathrm{~h}\right)$

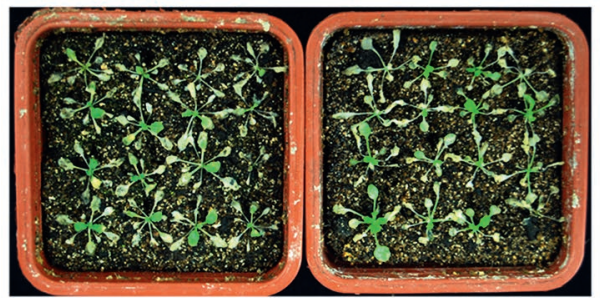

WT

B1L-OE \#1

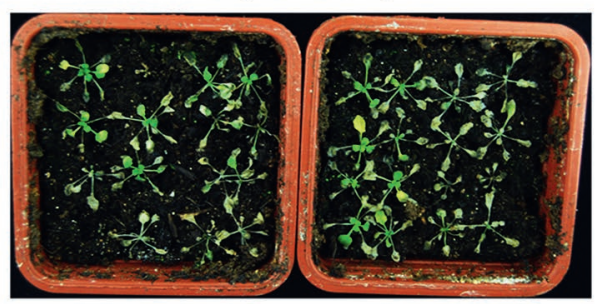

WT

B1L-OE \#1

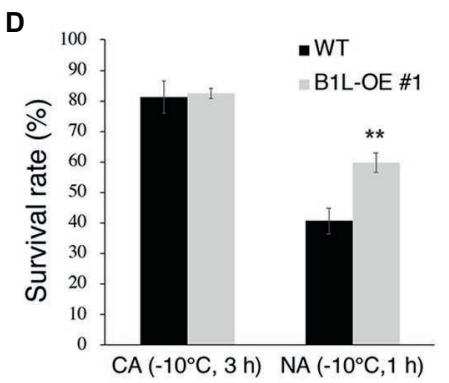

E $\mathrm{CA}\left(-10^{\circ} \mathrm{C}, 3 \mathrm{~h}\right)$

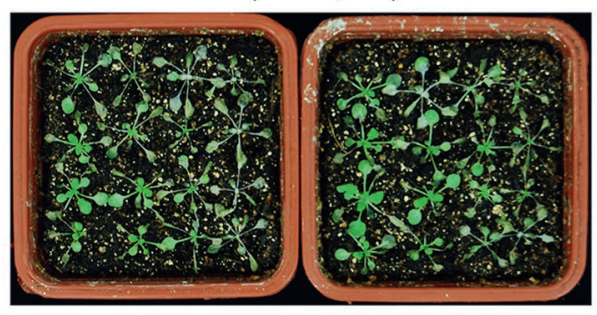

WT

B1L-OE \#2

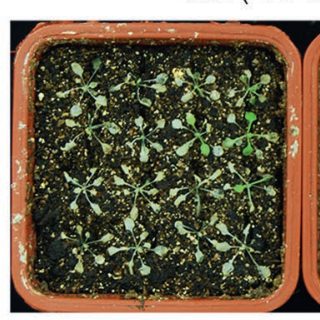

WT
$\mathrm{NA}\left(-10^{\circ} \mathrm{C}, 1 \mathrm{~h}\right)$

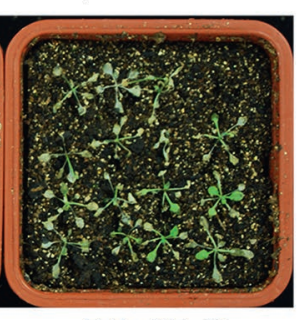

B1L-OE \#2
$\mathbf{F}$

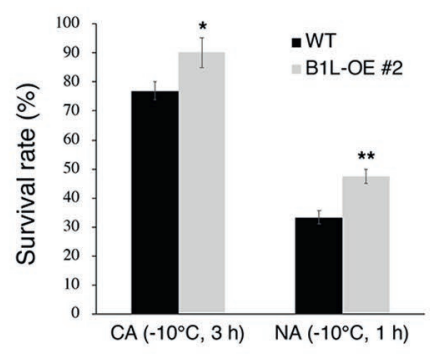

G

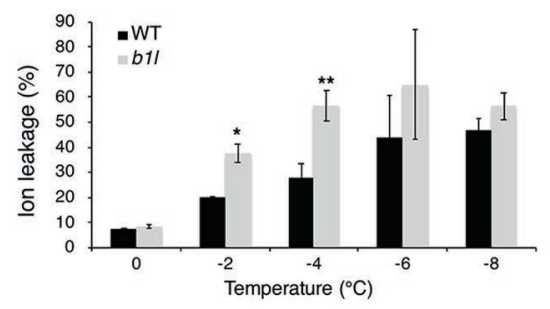

H

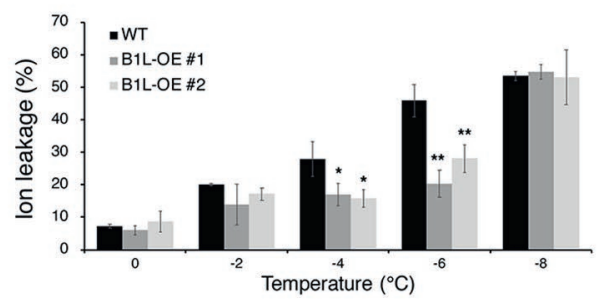

I

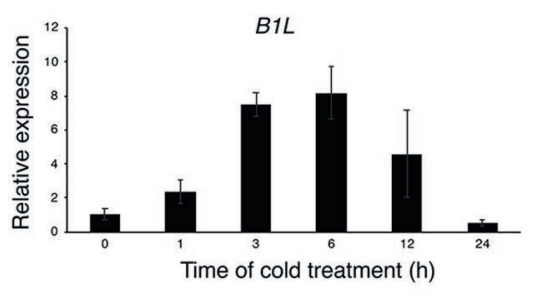

FIGURE 1 | B1L positively modulates freezing tolerance in Arabidopsis. (A-F) Freezing tolerance (A,C,E) and survival rates (B,D,F) of wild type (WT), b1/ mutant, and B1L-overexpressing plants under non-acclimated (NA) or cold-acclimated (CA) conditions. The 3-week-old plants were treated at -7 or $-10^{\circ} \mathrm{C}$ for $1 \mathrm{~h}(\mathrm{NA})$ or were pretreated at $4^{\circ} \mathrm{C}$ for 3 days and then treated at $-10^{\circ} \mathrm{C}$ for 1 or $3 \mathrm{~h}(\mathrm{CA})$. For each line, the survival rate assay was performed with four pots of 16 plants and scored 5 days later. The photos presented one pot of each line. The data are shown as means of three independent biological replicates \pm SD. Asterisks indicate significant differences $\left({ }^{*} p<0.05\right.$, and $\left.{ }^{* *} p<0.01\right)$ from wild type. $(\mathbf{G}, \mathbf{H})$ lon leakage of wild type, b1/ mutant, and B1L-overexpressing plants in $(\mathbf{A}, \mathbf{C}, \mathbf{E})$ after exposure to the temperature indicated. Data are means \pm SD. $n=4$ leaves, each from a different plant. Asterisks indicate significant differences ( ${ }^{*} p<0.05$ and $\left.{ }^{* *} p<0.01\right)$ from wild type. (I) Expression of B1L in wild type under cold treatment. Total RNA was extracted from 12-day-old seedlings treated at $4^{\circ} \mathrm{C}$ for $0,1,3,6,12,24 \mathrm{~h}$ and then subjected to qRT-PCR. Actin2/8 was used as a control. The expression of B1L in untreated wild type was set to 1 . The data are shown as means of three independent biological replicates \pm SD.

the candidate site, and Serine 213 in the N-terminal of B1L was identified (Figure 4C). This site was then mutated to alanine $\left(\mathrm{B} 1 \mathrm{~L}^{\mathrm{S} 213 \mathrm{~A}}\right)$ and aspartic acid $\left(\mathrm{B} 1 \mathrm{~L}^{\mathrm{S213 \textrm {D }}}\right)$ to mimic nonphosphorylation and autophosphorylation, respectively.
The $\mathrm{Y} 2 \mathrm{H}$ assays showed that both $\mathrm{B} 1 \mathrm{~L}^{\mathrm{S} 213 \mathrm{~A}}$ and $\mathrm{B} 1 \mathrm{~L}^{\text {S213D }}$ could

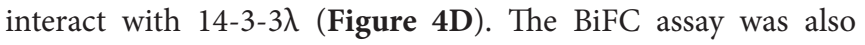
performed and showed the same results (Supplementary Figure S5). These results suggest that $\mathrm{B}_{1} \mathrm{~L}^{\mathrm{S} 213 \mathrm{~A}}$ and $\mathrm{B} 1 \mathrm{~L}^{\mathrm{S} 213 \mathrm{D}}$ 
A

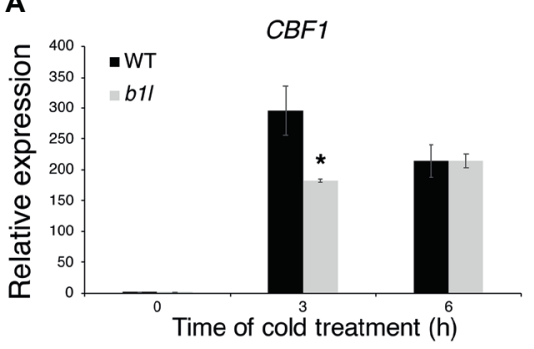

D

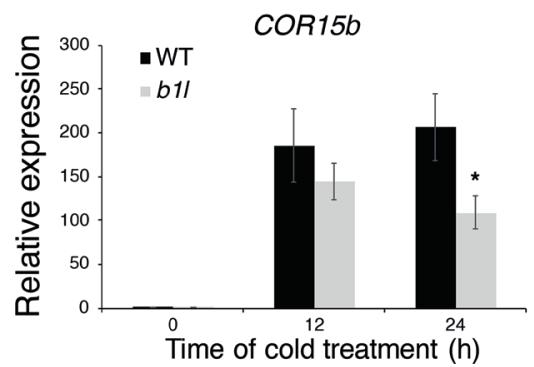

B

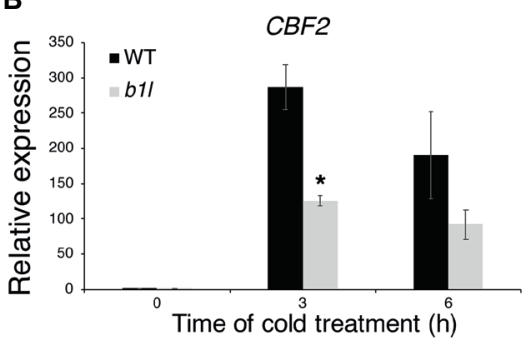

E

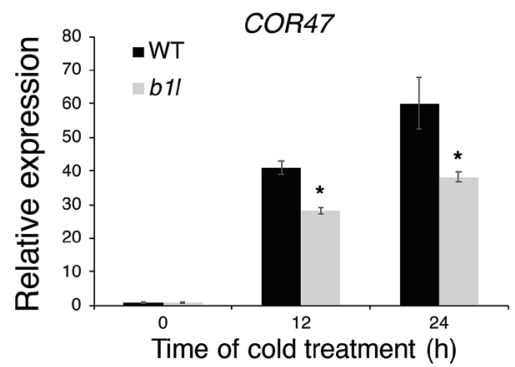

C

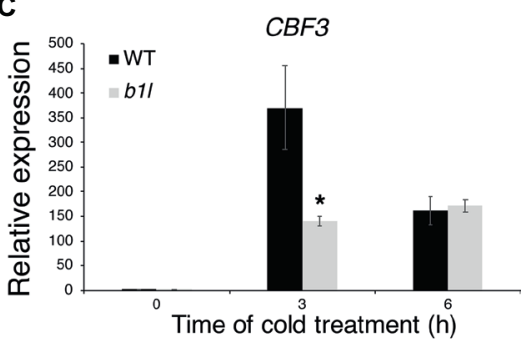

$\mathbf{F}$

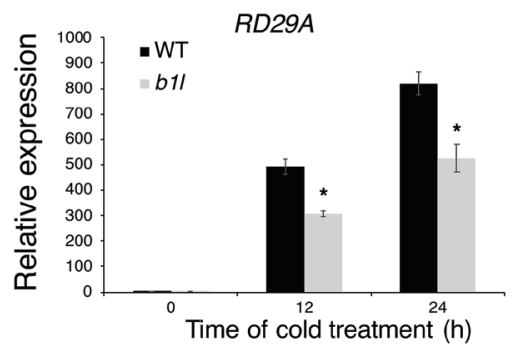

FIGURE 2 | B1L is a positive regulator of CBF pathway genes. The expression of CBF1, CBF2, and CBF3 (A-C) and CBF target genes COR15b, COR47, and $R D 29 A$ (D-F) in wild type and b1/ seedlings was determined via qRT-PCR analyses. Total RNA was extracted from 12 -day-old seedlings treated at $4{ }^{\circ} \mathrm{C}$ for 0,3 , $6 \mathrm{~h}$ for CBFs and 0, 12, $24 \mathrm{~h}$ for CBF target genes and then subjected to qRT-PCR. The data are shown as means of three independent biological replicates \pm SD. Asterisks indicate significant differences $\left({ }^{*} p<0.05\right)$ from wild type.

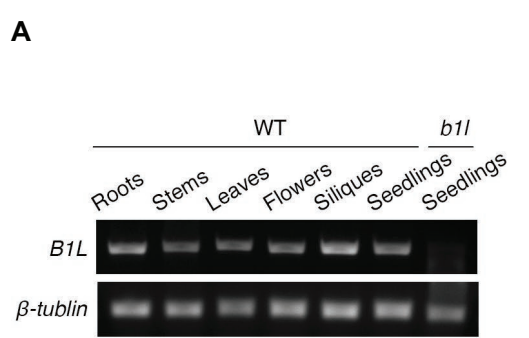

G

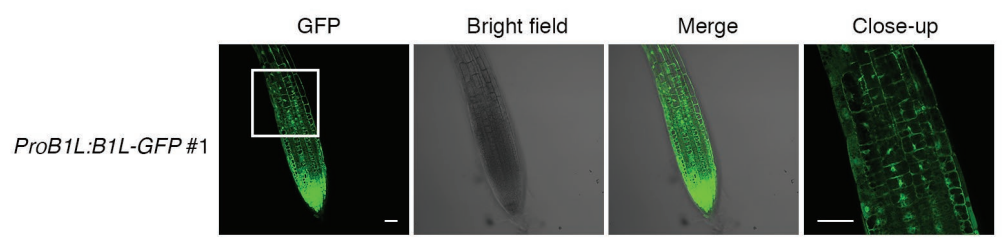

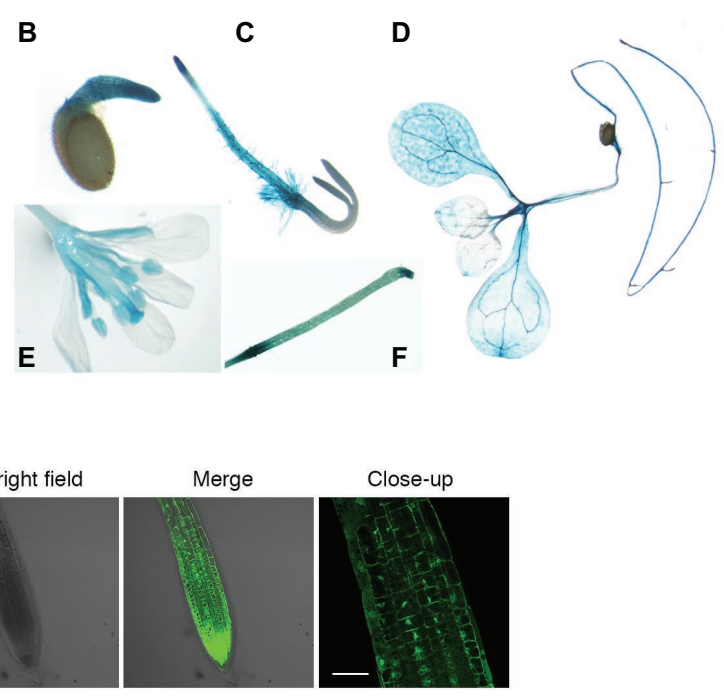

FIGURE 3 | The expression of B1L in different tissues and the subcellular localization of B1L. (A) RT-PCR analysis of B1L transcripts in the different tissues of wild type. The B1L transcript in the b1/ mutant seedlings was used as a negative control. $\beta$-TUBULIN was used as a loading control. (B-F) Histochemical analysis of the GUS reporter gene expression driven by the B1L promoter. The GUS signal was detected in 1-day-old seedlings (B), 2-day-old-seedlings (C), 10-day-old seedlings (D), and 8-week-old mature plants (E,F). (G) Localization of B1L in transgenic plants expressing B1L-GFP driven by its native promoter (ProB1L:B1L-GFP \#1). The signals in the root tips of 5-day-old plants were visualized. Bar $=50 \mu \mathrm{m}$.

may not be sufficient to affect the interaction between $\mathrm{B} 1 \mathrm{~L}$ and 14-3-3 $\lambda$. Then, the N-terminal (amino acids 1-238) and the C-terminal (amino acids 262-382) of B1L were used to identify the binding domain of B1L that interacts with 14-3-3 $\lambda$. The $\mathrm{Y} 2 \mathrm{H}$ assays showed that the $\mathrm{N}$-terminal of $\mathrm{B} 1 \mathrm{~L}$, but not the C-terminal of B1L, is sufficient for the interaction (Figure 4E).

\section{B1L Regulates Freezing Tolerance, Possibly Through a 14-3-3 $\lambda$-Dependent Pathway}

To understand the genetic interaction between B1L and 14-3-3 $\lambda$,

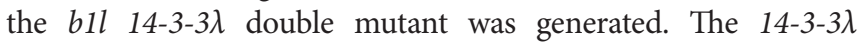
mutant displayed freezing tolerance similar to wild type 


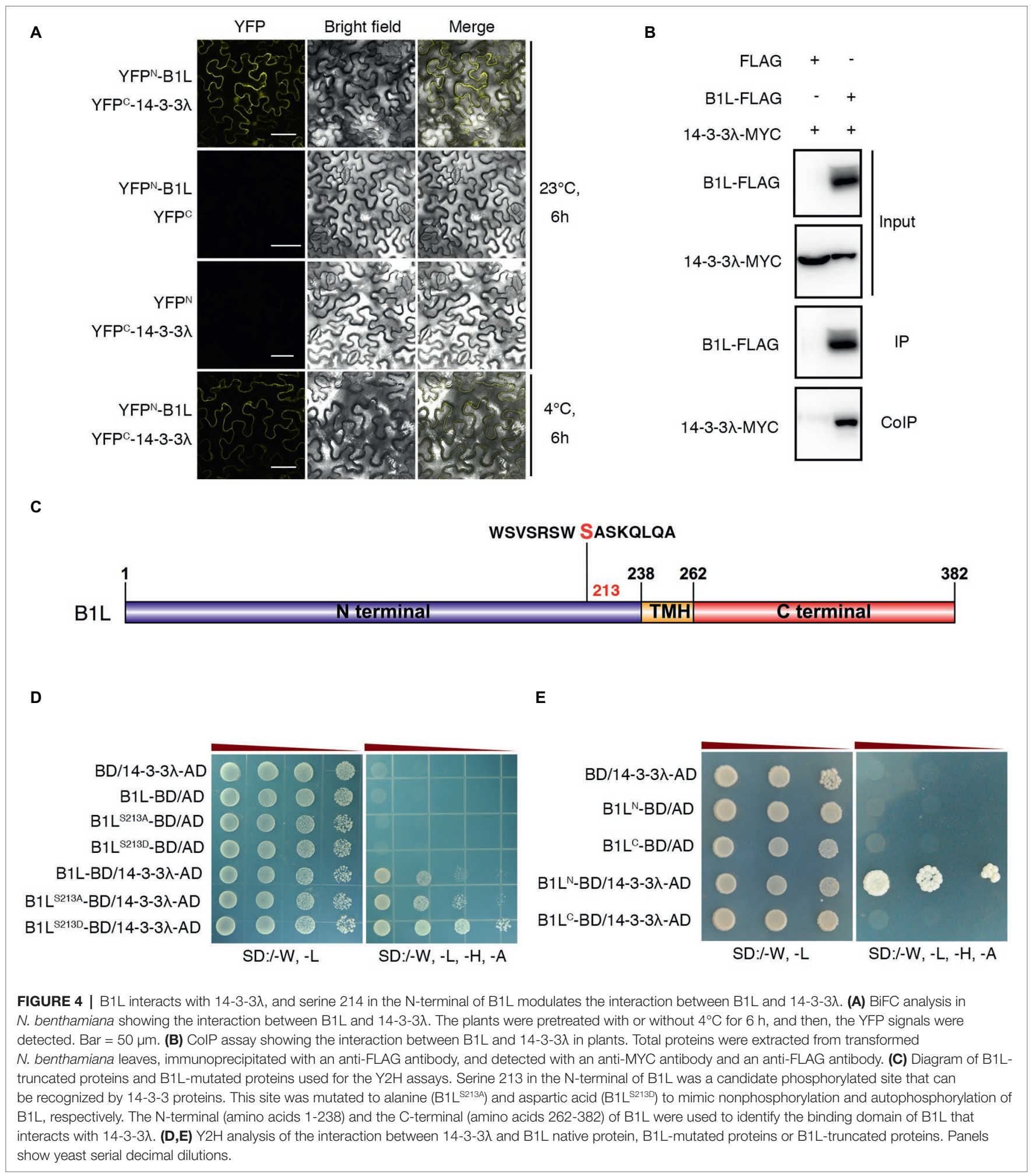

(Supplementary Figures S6A,B), consistent with a previous

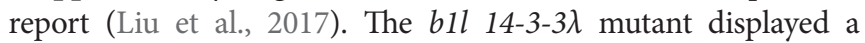
similar freezing sensitivity to $b 1 l$ (Supplementary Figures S6C,D). $14-3-3 \kappa$ is a close homologue to $14-3-3 \lambda$, and the $14-3-3 \kappa \lambda$ double mutant showed enhanced freezing tolerance in the previous report (Liu et al., 2017), as well as in our study (Figures 5A,B). A b1l $14-3-3 \kappa \lambda$ triple mutant was then generated, and this mutant showed enhanced freezing tolerance compared to that in wild type (Figures 5C,D). Consistently, the expression of $C O R$ genes was higher in $14-3-3 \kappa \lambda$ and b1l $14-3-3 \kappa \lambda$ plants 


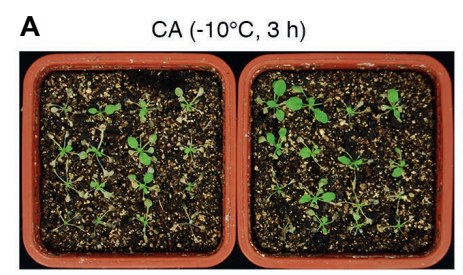

WT

C

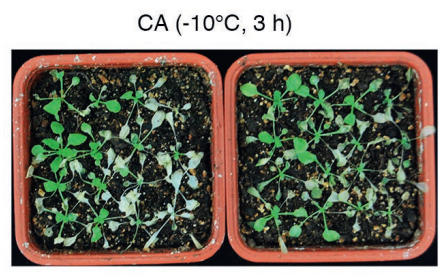

WT

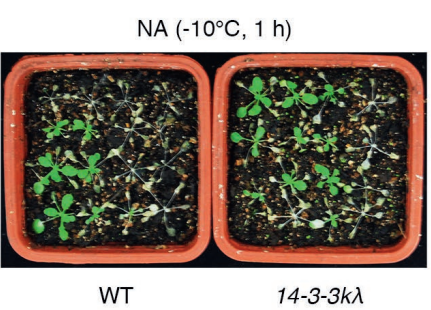

WT

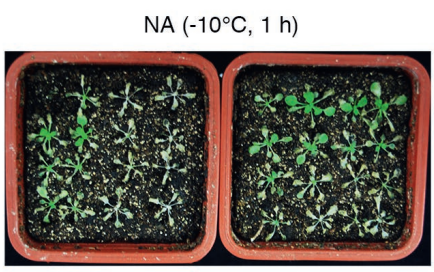

WT

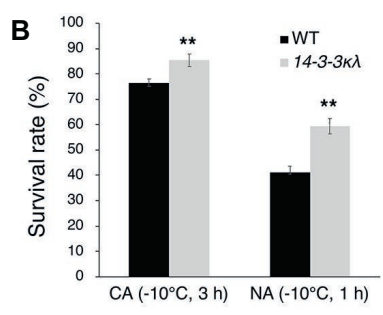

D

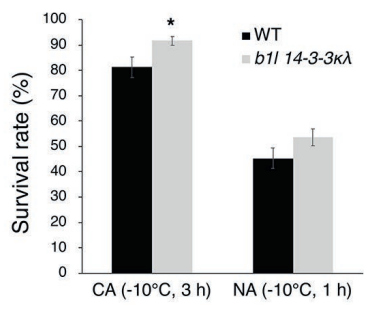

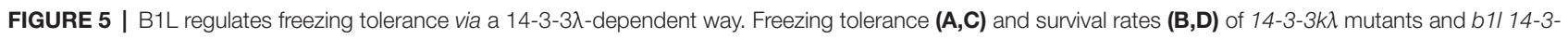
$3 k \lambda$ mutants under NA or CA conditions. The assays were performed as in Figure 1. The data are shown as means of three independent biological replicates \pm SD. Asterisks indicate significant differences $\left({ }^{*} p<0.05\right.$, and $\left.{ }^{* *} p<0.01\right)$ from wild type.

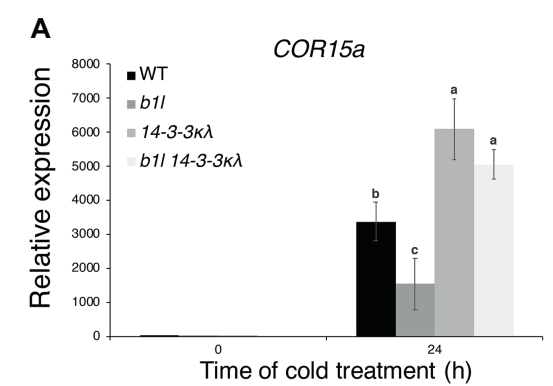

D

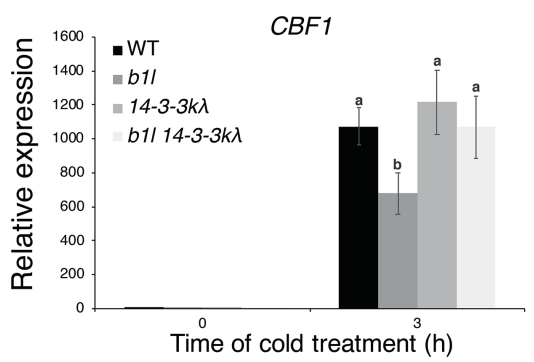

B

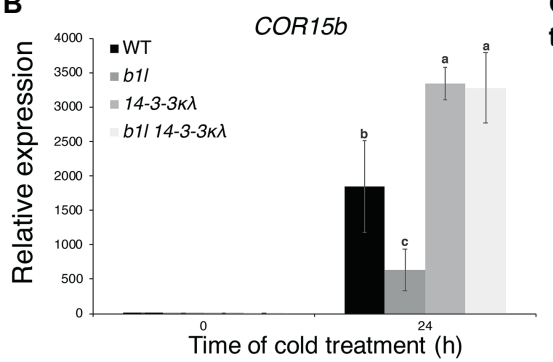

$\mathbf{E}$

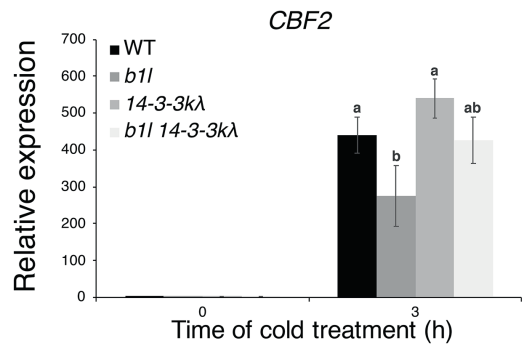

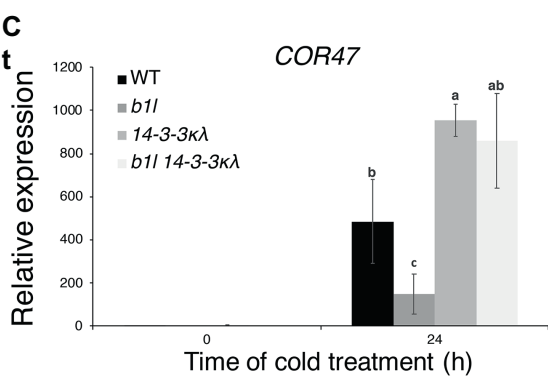

$\mathbf{F}$

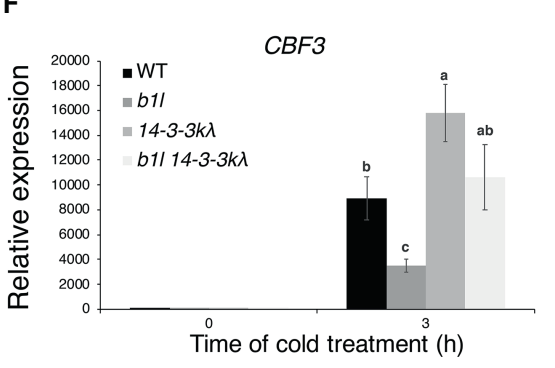

FIGURE 6 | The expression of CBFs and COR genes in 14-3-3k $\lambda$ and $b 1 / 14-3-3 k \lambda$ under cold treatment. The expression of the CBF target genes COR15a, COR15b, and COR47 (A-C) and CBF genes (D-F) in wild type, $b 11,14-3-3 k \lambda$, and $b 1 / 14-3-3 k \lambda$ seedlings was determined by qRT-PCR analyses. The data are shown as means of three independent biological replicates \pm SD. Significant differences $(p<0.05)$ are indicated by different lowercase letters.

than in wild type (Figures $\mathbf{6 A - C}$ ). Intriguingly, the expression of $C B F 3$ was significantly higher in $14-3-3 \kappa \lambda$ plants than in wild type (Figure 6F), which is consistent with the results from a previous study (Liu et al., 2017). qPCR results also showed that the reduced expression of all three $C B F$ genes in the $b 1 l$

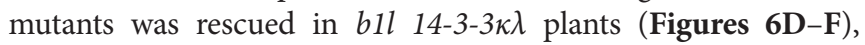
indicating that $\mathrm{B} 1 \mathrm{~L}$ and $14-3-3$ proteins may also participate in modulating the expression of CBFs. These results reveal that $\mathrm{B} 1 \mathrm{~L}$ regulates freezing tolerance possibly through $14-3-3 \lambda$.

\section{B1L Suppresses the Ubiquitin-Mediated Degradation of CBF3 via 14-3-3 $\lambda$}

The 14-3-3 proteins were shown to interact with and destabilize CBFs in the ubiquitin/26S proteasome pathway under cold stress (Liu et al., 2017). To verify whether B1L affects the degradation of $\mathrm{CBFs}$, several $\mathrm{CBF} 3$ degradation assays were performed.

First, an in vitro cell-free degradation assay was performed using purified CBF3-MBP proteins that were expressed in 


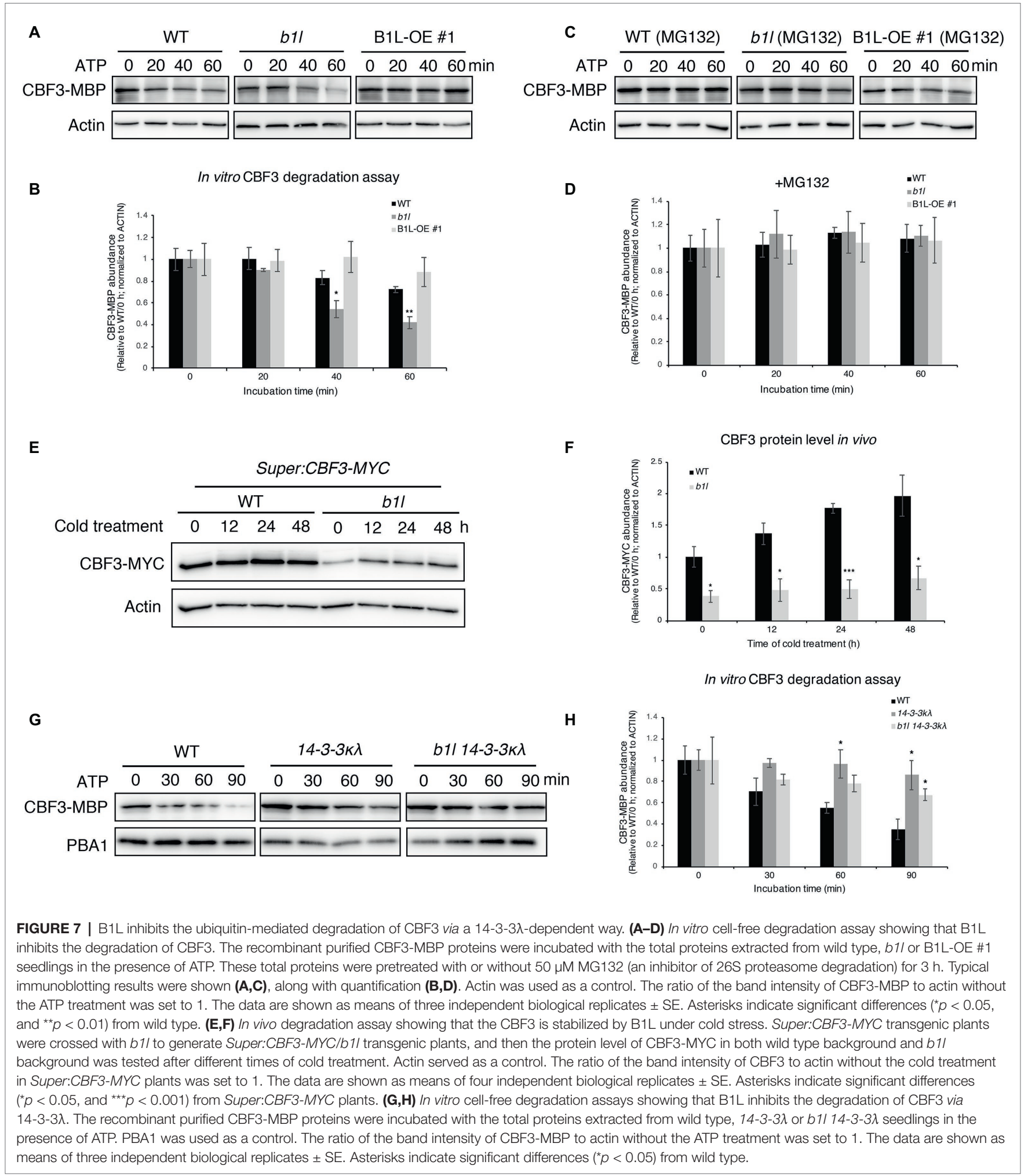

Escherichia coli. Western blotting results showed that the degradation of the CBF3-MBP increased when it was treated with the $b 1 l$ mutant total proteins; however, that the degradation of the CBF3-MBP was suppressed when it was treated with B1L-OE \#1 total proteins (Figures 7A,B). The degradation could be inhibited by MG132 (an inhibitor of 26S proteasome degradation) (Figures 7C,D). These results indicate that the stability of $\mathrm{CBF} 3$ is mediated by B1L.

Then, the protein level of $\mathrm{CBF} 3$ in vivo was examined. Super:CBF3-MYC transgenic plants (Liu et al., 2017) were 
A

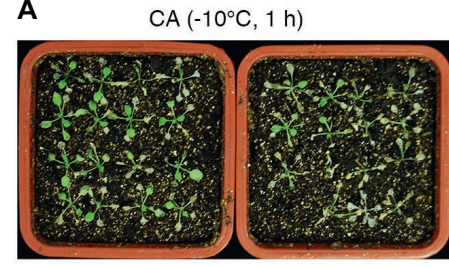

WT

C

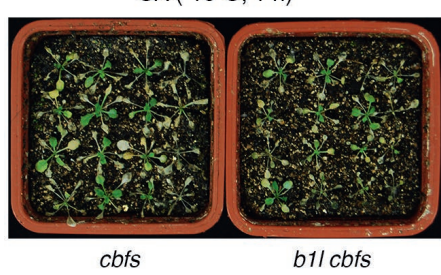

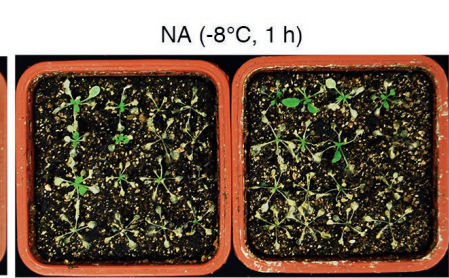

WT

cbfs

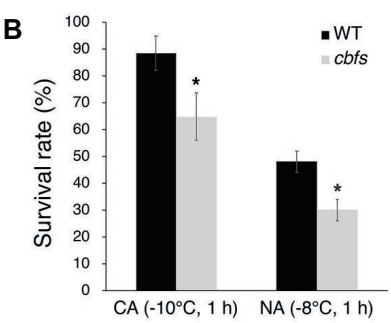

D
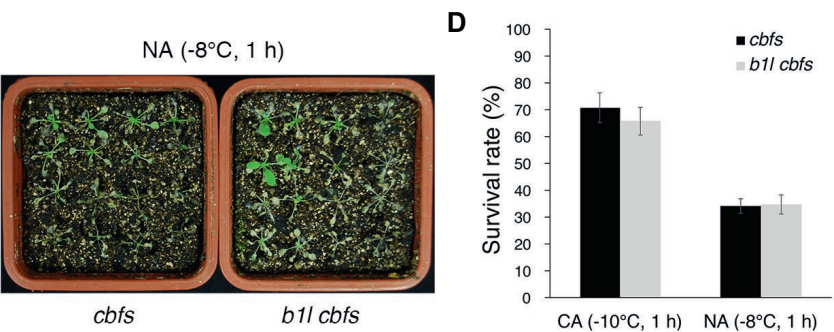

FIGURE 8 | B1L acts upstream of CBFs to regulate cold signaling. Freezing tolerance (A,C) and survival rates (B,D) of $c b f s$ mutants and $b 1 /$ cbfs mutants under NA or CA conditions. The assays were performed as in Figure 1. The data are shown as means of three independent biological replicates \pm SD. Asterisks indicate significant differences $\left({ }^{*} p<0.05\right)$ from wild type.

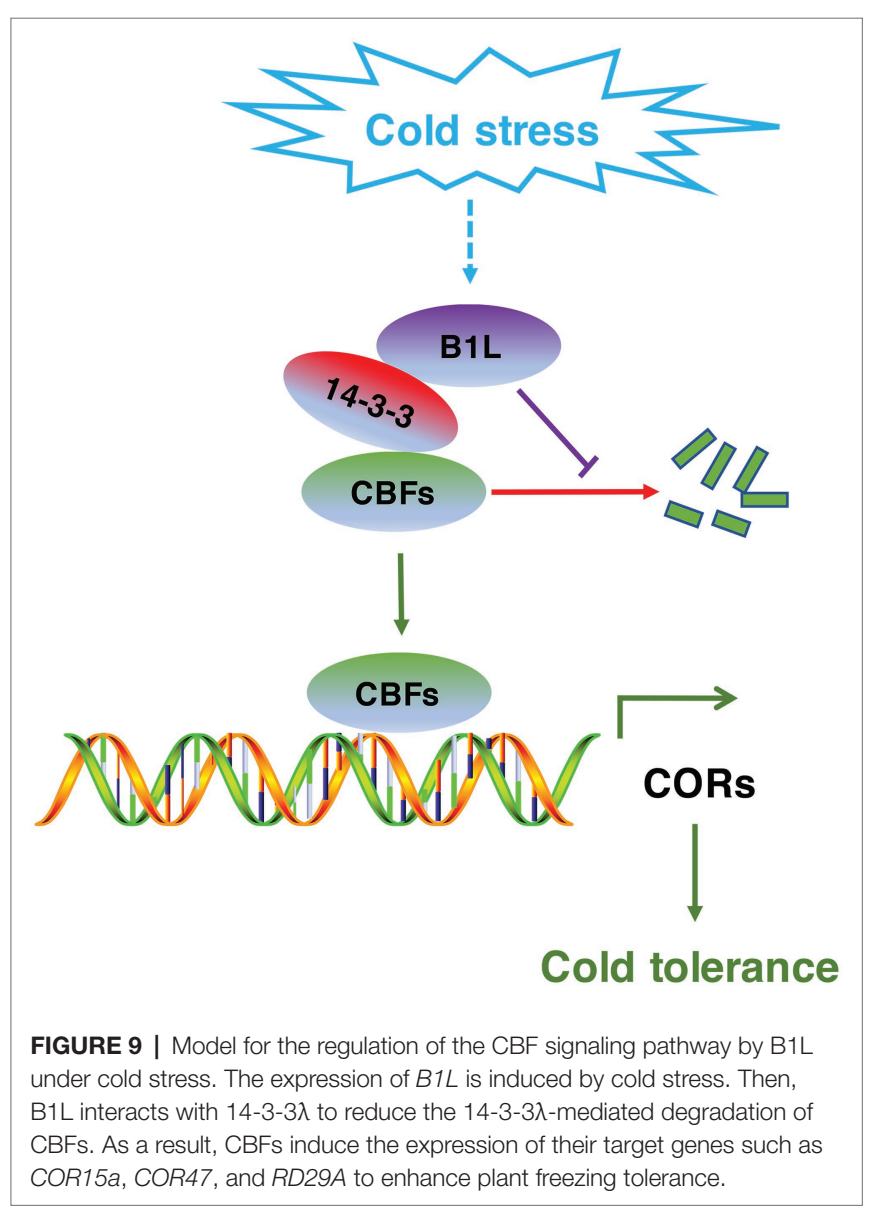

crossed with $b 1 l$, and the protein levels of CBF3-MYC in both wild type background and $b 1 l$ background were analyzed after different time of cold treatment. The protein level of CBF3-MYC was much lower in $b 1 l$ mutants than wild type before and after cold treatment $\left(4^{\circ} \mathrm{C}\right)$ (Figures 7E,F). These results further reveal that the stability of $\mathrm{CBF} 3$ is mediated by B1L.

To explore the role of 14-3-3 proteins in the B1L-mediated degradation of $\mathrm{CBFs}$, the purified $\mathrm{CBF} 3-\mathrm{MBP}$ proteins were

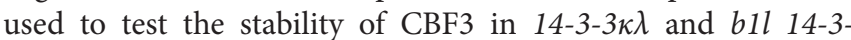
$3 \kappa \lambda$ mutants. Western blotting results showed that the $14-3-3 \kappa \lambda$ mutant suppressed the degradation of CBF3-MBP compared to wild type (Figures $\mathbf{7 G}, \mathbf{H}$ ), as previously reported (Liu et al.,

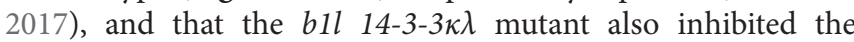
degradation of the CBF3 (Figures 7G,H). These results show

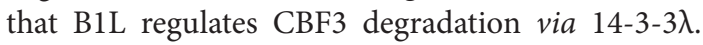

\section{B1L Acts Upstream of CBFs, Positively Regulating Plant Freezing Tolerance}

To further examine the relationship between B1L and CBFs, b1l mutant was crossed with cbfs triple mutant (Jia et al., 2016). The $c b f s$ mutant was more sensitive to freezing treatment than wild type (Figures 8A,B), consistent with previous studies (Jia et al., 2016; Liu et al., 2017). The b1l cbfs quadruple mutant did not aggravate the freezing sensitivity of $c b f s$ (Figures 8C,D). These results further indicate that B1L positively regulating plant freezing tolerance via $\mathrm{CBF}$ proteins.

\section{DISCUSSION}

CBF signaling pathway has important roles in cold acclimation. The expression of $C B F s$ is regulated by numerous transcription factors. However, studies about the posttranslational regulation of CBFs are limited. Here, we show that B1L participates in regulating freezing tolerance partly through repressing the degradation of CBFs (Figure 9). Several lines of evidence were provided: (1) B1L promotes the expression of CBF pathway 
genes and therefore freezing tolerance in Arabidopsis. (2) B1L directly interacts with $14-3-3 \lambda$. (3) B1L inhibits the 14-3-3 protein-mediated degradation of CBFs. (4) The $b 1 l c b f s$ quadruple mutant displayed a freezing sensitivity similar to $c b f s$.

The 14-3-3 proteins serve important roles in many processes, such as stomata movements, phytohormone regulation, biotic stress, and abiotic stress (Cotelle et al., 2000; Gampala et al., 2007; Wang et al., 2011; de Boer et al., 2013; Catala et al., 2014; Kaundal et al., 2017; Keicher et al., 2017; Liu et al., 2017). These proteins mostly interact with phosphorylated proteins and affect the subcellular localization, protein stability, enzymatic activity of target proteins or the interaction between the target proteins and other proteins (Jaspert et al., 2011; Wang et al., 2011; Yoon and Kieber, 2013; Zhou et al., 2014; Huang et al.,

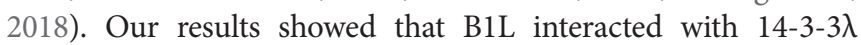
(Figure 4), indicating that $\mathrm{B} 1 \mathrm{~L}$ may also be a phosphorylated protein. Serine 213 in the N-terminal of B1L is a potential phosphorylated site that can be recognized by 14-3-3 proteins (Figure 4C), and the N-terminal of B1L is sufficient for the interaction between B1L and 14-3-3 $\lambda$. However, mutating Serine 213 to alanine $\left(B 1 \mathrm{~L}^{\mathrm{S} 213 \mathrm{~A}}\right)$ did not abolish the interaction between $\mathrm{B} 1 \mathrm{~L}$ and $14-3-3 \lambda$, indicating the possible involvement of new sites to affect the interaction between B1L and 14-3-3 $\lambda$ (Figure 4D; Supplementary Figure S5). The phosphorylated site of B1L that affects the interaction between B1L and $14-3-3 \lambda$ and the biological roles of these sites need to be further investigated.

The 14-3-3 protein 14-3-3\% (also known as RARE COLD INDUCIBLE $1 \mathrm{~A}$ or GRF3) has also been shown to participate in the regulation of freezing tolerance via an ethylene biosynthesis pathway (Catala et al., 2014). It interacts with ACS6 and inhibits the expression of ethylene-related genes and $C B F$ s, leading to decreased freezing tolerance (Catala et al., 2014). Although the expression of CBFs was reduced in b1l mutants under cold treatment (Figure 2), the expression of ethylene-related genes (ERF4 and ERF11) was not significantly changed in b1l mutants compare to wild type (Supplementary Figures S7A,B). The interaction between B1L and 14-3-3 $\psi$ was also not observed in the $\mathrm{Y} 2 \mathrm{H}$ system (Supplementary Figure S7C). These results indicate that B1L may specifically interact with $14-3-3 \lambda$, modulating freezing tolerance via an ethylene-independent pathway.

The stability of CBFs has been shown to be modulated by 14-3-3 proteins and BTF3L via a ubiquitin/26S proteasome pathway (Liu et al., 2017; Ding et al., 2018). HOS15, which is an E3 ligase, interacts with $\mathrm{CBF}$, affecting the expression of COR genes but does not participate in the regulation of $\mathrm{CBF}$ degradation (Park et al., 2018). Therefore, the components of the ubiquitin/26S proteasome that participate in degrading CBFs are still unknown. More proteins that are involved in the ubiquitin/26S proteasome pathway need to be identified to elucidate the mechanism of CBF stability. Our protein degradation assay in vivo and in vitro both showed that B1L affects the stability of CBF (Figures 7A-D), indicating that B1L is another regulator that participate in the regulation of $\mathrm{CBF}$ proteins.

The overexpression of $C B F$ s leads to dwarfism and late-flowering (Jaglo-Ottosen, 1998; Achard et al., 2008; Zhou et al., 2017). ICE1 is a positive regulator of $C B F$ s, and its overexpressing lines result in late-flowering, due to ICE1 directly binding to the promoter of FLC and inducing the expression of the FLC (Lee et al., 2015). Therefore, plants fine-tune CBF signaling pathway to avoid harmful effects during their development. To be specific, 14-3-3 $\lambda$ can be translocated to the nucleus under cold conditions, reducing the abundance of CBFs (Liu et al., 2017). Cold treatment induces the expression of $B 1 L$ but does not obviously change the subcellular localization of B1L (Supplementary Figure S8). We hypothesis that the interaction between $14-3-3 \lambda$ and B1L may lead to a delay in the nuclear translocation of $14-3-3 \lambda$ after cold treatment. This translocation delay in turn allows plants to fine-tune the abundance of CBFs to adapt to cold stress.

\section{DATA AVAILABILITY}

All datasets for this study are included in the manuscript and/ or the Supplementary Files.

\section{AUTHOR CONTRIBUTIONS}

TC, J-HC, WZ, GY, and L-JY performed the experiments. TC analyzed the data. TC, HZ, and L-ZA designed the project and drafted the manuscript. D-ML, BL, and H-MS revised the manuscript critically for important intellectual content. HZ and L-ZA supervised the project and complemented the writing.

\section{FUNDING}

This work was supported by the Key Program National Natural Science Foundation of China (41830321); the National Natural Science Foundation of China (31770432, 31570488, 31301078); the National Basic Research Program of China (973 Program) (2013CB429904); the Science and Technology Partnership Program, Ministry of Science and Technology of China (KY201501008); the National Science and Technology Major Project in Gansu (17ZD2WA017); and the Ministry of Agriculture of the People's Republic of China (2016ZX08009-003-002).

\section{ACKNOWLEDGMENTS}

We thank Jia Li and Xiao-Ping Gou (Lanzhou University) as well as Shu-Hua Yang (China Agricultural University) for kindly providing Arabidopsis seeds. We thank Sui-Wen Hou (Lanzhou University) for valuable comments on the manuscript. We are grateful to Xiu-Le Yue and the Core Facility for Life Science Research (Lanzhou University) for technical assistance.

\section{SUPPLEMENTARY MATERIAL}

The Supplementary Material for this article can be found online at: https://www.frontiersin.org/articles/10.3389/fpls.2019.00807/ full\#supplementary-material 


\section{REFERENCES}

Achard, P., Gong, F., Cheminant, S., Alioua, M., Hedden, P., and Genschik, P. (2008). The cold-inducible CBF1 factor-dependent signaling pathway modulates the accumulation of the growth-repressing DELLA proteins via its effect on gibberellin metabolism. Plant Cell 20, 2117-2129. doi: 10.1105/ tpc. 108.058941

Agarwal, M., Hao, Y., Kapoor, A., Dong, C. H., Fujii, H., Zheng, X., et al. (2006). A R2R3 type MYB transcription factor is involved in the cold regulation of $C B F$ genes and in acquired freezing tolerance. J. Biol. Chem. 281, 37636-37645. doi: 10.1074/jbc.M605895200

Catala, R., Lopez-Cobollo, R., Mar Castellano, M., Angosto, T., Alonso, J. M., Ecker, J. R., et al. (2014). The Arabidopsis 14-3-3 protein rare cold inducible 1A links low-temperature response and ethylene biosynthesis to regulate freezing tolerance and cold acclimation. Plant Cell 26, 3326-3342. doi: 10.1105/tpc.114.127605

Chen, L., Guan, L., Qian, P., Xu, F., Wu, Z., Wu, Y., et al. (2016). NRPB3, the third largest subunit of RNA polymerase II, is essential for stomatal patterning and differentiation in Arabidopsis. Development 143, 1600-1611. doi: 10.1242/dev.129098

Chinnusamy, V., Ohta, M., Kanrar, S., Lee, B., Hong, X., Agarwal, M., et al. (2003). ICE1: a regulator of cold-induced transcriptome and freezing tolerance in Arabidopsis. Genes Dev. 17, 1043-1054. doi: 10.1101/gad.1077503

Cotelle, V., Meek, S. E., Provan, F., Milne, F. C., Morrice, N., and MacKintosh, C. (2000). 14-3-3s regulate global cleavage of their diverse binding partners in sugar-starved Arabidopsis cells. EMBO J. 19, 2869-2876. doi: 10.1093/ emboj/19.12.2869

de Boer, A. H., van Kleeff, P. J. M. M., and Gao, J. (2013). Plant 14-3-3 proteins as spiders in a web of phosphorylation. Protoplasma 250, 425-440. doi: 10.1007/s00709-012-0437-z

Ding, Y., Jia, Y., Shi, Y., Zhang, X., Song, C., Gong, Z., et al. (2018). OST1mediated BTF3L phosphorylation positively regulates $\mathrm{CBFs}$ during plant cold responses. EMBO J. 37:e98228. doi: 10.15252/embj.201798228

Doherty, C. J., Van Buskirk, H. A., Myers, S. J., and Thomashow, M. F. (2009). Roles for Arabidopsis CAMTA transcription factors in cold-regulated gene expression and freezing tolerance. Plant Cell 21, 972-984. doi: 10.1105/ tpc. 108.063958

Dong, M. A., Farre, E. M., and Thomashow, M. F. (2011). Circadian clockassociated 1 and late elongated hypocotyl regulate expression of the C-repeat binding factor (CBF) pathway in Arabidopsis. Proc. Natl. Acad. Sci. USA 108, 7241-7246. doi: 10.1073/pnas.1103741108

Gampala, S. S., Kim, T. W., He, J. X., Tang, W., Deng, Z., Bai, M. Y., et al. (2007). An essential role for 14-3-3 proteins in brassinosteroid signal transduction in Arabidopsis. Dev. Cell 13, 177-189. doi: 10.1016/j. devcel.2007.06.009

Gilmour, S. J., Zarka, D. G., Stockinger, E. J., Salazar, M. P., Houghton, J. M., and Thomashow, M. F. (1998). Low temperature regulation of the Arabidopsis CBF family of AP2 transcriptional activators as an early step in cold-induced COR gene expression. Plant J. 16, 433-442. doi: 10.1046/j.1365-313x.1998.00310.x

Guy, C. L. (1990). Cold acclimation and freezing stress tolerance: role of protein metabolism. Annu. Rev. Plant Physiol. Plant Mol. Biol. 41, 187-223.

Huang, X., Zhang, Q., Jiang, Y., Yang, C., Wang, Q., and Li, L. (2018). Shadeinduced nuclear localization of PIF7 is regulated by phosphorylation and 14-3-3 proteins in Arabidopsis. elife 7, 470-476. doi: 10.7554/eLife.31636

Jaglo-Ottosen, K. R. (1998). Arabidopsis CBF1 overexpression induces COR genes and enhances freezing tolerance. Science 280, 104-106. doi: 10.1126/ science.280.5360.104

Jaspert, N., Throm, C., and Oecking, C. (2011). Arabidopsis 14-3-3 proteins: fascinating and less fascinating aspects. Front. Plant Sci. 2:96. doi: 10.3389/ fpls.2011.00096

Jia, Y., Ding, Y., Shi, Y., Zhang, X., Gong, Z., and Yang, S. (2016). The $c b f s$ triple mutants reveal the essential functions of CBFs in cold acclimation and allow the definition of CBF regulons in Arabidopsis. New Phytol. 212, 345-353. doi: 10.1111/nph.14088

Jiang, B., Shi, Y., Zhang, X., Xin, X., Qi, L., Guo, H., et al. (2017). PIF3 is a negative regulator of the $C B F$ pathway and freezing tolerance in Arabidopsis. Proc. Natl. Acad. Sci. USA 114, E6695-E6702. doi: 10.1073/ pnas. 1706226114
Kaundal, A., Ramu, V. S., Oh, S., Lee, S., Pant, B. D., Lee, H.-K., et al. (2017). General control nonrepressible4 (GCN4) degrades 14-3-3 and the RIN4 complex to regulate stomatal aperture with implications on nonhost disease resistance and drought tolerance. Plant Cell 29, 2233-2248. doi: 10.1105/tpc.17.00070

Keicher, J., Jaspert, N., Weckermann, K., Möller, C., Throm, C., Kintzi, A., et al. (2017). Arabidopsis 14-3-3 epsilon members contribute to polarity of PIN auxin carrier and auxin transport-related development. elife 6, 1-21. doi: $10.7554 /$ eLife.24336.001

Kulich, I., Cole, R., Drdová, E., Cvrčková, F., Soukup, A., Fowler, J., et al. (2010). Arabidopsis exocyst subunits SEC8 and EXO70A1 and exocyst interactor ROH1 are involved in the localized deposition of seed coat pectin. New Phytol. 188, 615-625. doi: 10.1111/j.1469-8137.2010.03372.x

Lee, J. H., Jung, J. H., and Park, C. M. (2015). Inducer of CBF expression 1 integrates cold signals into flowering locus $\mathrm{C}$-mediated flowering pathways in Arabidopsis. Plant J. 84, 29-40. doi: 10.1111/tpj.12956

Lee, D.-K., Parrott, D. L., Adhikari, E., Fraser, N., and Sieburth, L. E. (2016). The mobile bypass signal arrests shoot growth by disrupting shoot apical meristem maintenance, cytokinin signaling, and WUS transcription factor expression. Plant Physiol. 171, 2178-2190. doi: 10.1104/pp.16.00474

Li, H., Ding, Y., Shi, Y., Zhang, X., Zhang, S., Gong, Z., et al. (2017a). MPK3and MPK6-mediated ICE1 phosphorylation negatively regulates ICE1 stability and freezing tolerance in Arabidopsis. Dev. Cell 43, 630-642. doi: 10.1016/j. devcel.2017.09.025

Li, H., Ye, K., Shi, Y., Cheng, J., Zhang, X., and Yang, S. (2017b). BZR1 positively regulates freezing tolerance via $\mathrm{CBF}$-dependent and $\mathrm{CBF}$-independent pathways in Arabidopsis. Mol. Plant 10, 545-559. doi: 10.1016/j.molp.2017.01.004

Liu, Z., Jia, Y., Ding, Y., Shi, Y., Li, Z., Guo, Y., et al. (2017). Plasma membrane CRPK1-mediated phosphorylation of 14-3-3 proteins induces their nuclear import to fine-tune CBF signaling during cold response. Mol. Cell 66, 117-128.e5. doi: 10.1016/j.molcel.2017.02.016

Liu, Q., Kasuga, M., Sakuma, Y., Abe, H., Miura, S., Yamaguchi-Shinozaki, K., et al. (1998). Two transcription factors, DREB1 and DREB2, with an EREBP/ AP2 DNA binding domain separate two cellular signal transduction pathways in drought- and low-temperature-responsive gene expression, respectively, in Arabidopsis. Plant Cell 10, 1391-1406. doi: 10.1105/tpc.10.8.1391

Liu, L., Zhang, Y., Tang, S., Zhao, Q., Zhang, Z., Zhang, H., et al. (2010). An efficient system to detect protein ubiquitination by agroinfiltration in Nicotiana benthamiana. Plant J. 61, 893-903. doi: 10.1111/j.1365-313X.2009.04109.x

Ma, S., and Bohnert, H. J. (2007). Integration of Arabidopsis thaliana stressrelated transcript profiles, promoter structures, and cell-specific expression. Genome Biol. 8:R49. doi: 10.1186/gb-2007-8-4-r49

Miura, K., Jin, J. B., Lee, J., Yoo, C. Y., Stirm, V., Miura, T., et al. (2007). SIZ1-mediated sumoylation of ICE1 controls CBF3/DREB1A expression and freezing tolerance in Arabidopsis. Plant Cell 19, 1403-1414. doi: 10.1105/ tpc.106.048397

Park, J., Lim, C. J., Shen, M., Park, H. J., Cha, J.-Y., Iniesto, E., et al. (2018). Epigenetic switch from repressive to permissive chromatin in response to cold stress. Proc. Natl. Acad. Sci. USA 115, E5400-E5409. doi: 10.1073/ pnas. 1721241115

Shi, Y., Tian, S., Hou, L., Huang, X., Zhang, X., Guo, H., et al. (2012). Ethylene signaling negatively regulates freezing tolerance by repressing expression of CBF and type-A ARR genes in Arabidopsis. Plant Cell 24, 2578-2595. doi: $10.1105 /$ tpc. 112.098640

Stockinger, E. J., Gilmour, S. J., and Thomashow, M. F. (1997). Arabidopsis thaliana CBF1 encodes an AP2 domain-containing transcriptional activator that binds to the C-repeat/DRE, a cis-acting DNA regulatory element that stimulates transcription in response to low temperature and water deficit. Proc. Natl. Acad. Sci. USA 94, 1035-1040. doi: 10.1073/pnas.94.3.1035

Thomashow, M. F. (1999). Plant cold acclimation: freezing tolerance genes and regulatory mechanisms. Annu. Rev. Plant Physiol. Plant Mol. Biol. 50, 571-599. doi: 10.1146/annurev.arplant.50.1.571

Van Kleeff, P. J., Jaspert, N., Li, K. W., Rauch, S., Oecking, C., and de Boer, A. H. (2014). Higher order Arabidopsis 14-3-3 mutants show 14-3-3 involvement in primary root growth both under control and abiotic stress conditions. J. Exp. Bot. 65, 5877-5888. doi: 10.1093/jxb/eru338

Van Norman, J. M., Frederick, R. L., and Sieburth, L. E. (2004). BYPASS1 negatively regulates a root-derived signal that controls plant architecture. Curr. Biol. 14, 1739-1746. doi: 10.1016/j.cub.2004.09.045 
Van Norman, J. M., Murphy, C., and Sieburth, L. E. (2011). BYPASS1: synthesis of the mobile root-derived signal requires active root growth and arrests early leaf development. BMC Plant Biol. 11:28. doi: 10.1186/1471-2229-11-28

Visscher, A. M., Belfield, E. J., Vlad, D., Irani, N., Moore, I., and Harberd, N. P. (2015). Overexpressing the multiple-stress responsive gene Atlg74450 reduces plant height and male fertility in Arabidopsis thaliana. PLoS One 10:e0140368. doi: 10.1371/journal.pone.0140368

Wang, H., Yang, C., Zhang, C., Wang, N., Lu, D., Wang, J., et al. (2011). Dual role of BKI1 and 14-3-3 s in Brassinosteroid signaling to link receptor with transcription factors. Dev. Cell 21, 825-834. doi: 10.1016/j.devcel.2011.08.018

Wang, F., Zhu, D., Huang, X., Li, S., Gong, Y., Yao, Q., et al. (2009). Biochemical insights on degradation of Arabidopsis DELLA proteins gained from a cellfree assay system. Plant Cell Online 21, 2378-2390. doi: 10.1105/tpc.108.065433

Yamaguchi-Shinozaki, K., and Shinozaki, K. (1994). A novel cis-Acting element in an Arabidopsis gene is involved in responsiveness to drought, lowtemperature, or high-salt stress. Plant Cell 6, 251-264. doi: 10.1105/tpc.6.2.251

Yoon, G. M., and Kieber, J. J. (2013). 14-3-3 regulates 1-aminocyclopropane-1carboxylate synthase protein turnover in Arabidopsis. Plant Cell 25, 1016-1028. doi: 10.1105/tpc.113.110106

Zhao, C., Wang, P., Si, T., Hsu, C.-C., Wang, L., Zayed, O., et al. (2017). MAP kinase cascades regulate the cold response by modulating ICE1 protein stability. Dev. Cell 43, 1-12. doi: 10.1016/j.devcel.2017.09.024
Zhou, M., Chen, H., Wei, D., Ma, H., and Lin, J. (2017). Arabidopsis CBF3 and DELLAs positively regulate each other in response to low temperature. Sci. Rep. 7:39819. doi: 10.1038/srep39819

Zhou, H., Lin, H., Chen, S., Becker, K., Yang, Y., Zhao, J., et al. (2014). Inhibition of the Arabidopsis salt overly sensitive pathway by 14-3-3 proteins. Plant Cell 26, 1166-1182. doi: 10.1105/tpc.113.117069

Zhu, J., Shi, H., Lee, B. -h., Damsz, B., Cheng, S., Stirm, V., et al. (2004). An Arabidopsis homeodomain transcription factor gene, HOS9, mediates cold tolerance through a CBF-independent pathway. Proc. Natl. Acad. Sci. USA 101, 9873-9878. doi: 10.1073/pnas.0403166101

Conflict of Interest Statement: The authors declare that the research was conducted in the absence of any commercial or financial relationships that could be construed as a potential conflict of interest.

Copyright (c) 2019 Chen, Chen, Zhang, Yang, Yu, Li, Li, Sheng, Zhang and An. This is an open-access article distributed under the terms of the Creative Commons Attribution License (CC BY). The use, distribution or reproduction in other forums is permitted, provided the original author(s) and the copyright owner(s) are credited and that the original publication in this journal is cited, in accordance with accepted academic practice. No use, distribution or reproduction is permitted which does not comply with these terms. 